\title{
The combined impacts of docosahexaenoic acid, endurance physical exercise, and prolonged fasting on brain function
}

\author{
Yi-Sub Kwak', Sun Young Lim²* \\ 'Department of Physical Education, College of Arts, Design and Sports Science, Dong-Eui University, Busan, Korea \\ ${ }^{2}$ Division of Marine Bioscience, Korea Maritime and Ocean University, Busan, Korea
}

Docosahexaenoic acid (DHA), a long chain polyunsaturated fatty acid (PUFA) is highly enriched in the membrane phospholipids of the brain, neuronal tissue and retina. Accumulating evidence suggests that brain DHA is necessary for maintaining for optimal function of the cognition and the visual system, particularly the photoreceptor, the retina. Loss of brain DHA, especially during crucial brain development period, was highly associated with visual and cognitive defects. Here it would be addressed the effect of DHA on brain functions as assessed by spatial task performance using a first generation model which is similar to human nutrition case. And also, it was well known that prolonged fasting and energy restriction with endurance physical exercise stimulate cognitive function and brain function, which is caused by upregulated ke- tone body and upregulated brain-derived neurotropic factor (BDNF) responses. In the DHA intake or prolonged physical exercise, upregulated BDNF can activate mitochondrial biogenesis to elevate neuronal bioenergetics and enable synaptic formation. And it also can activate DNA repair in neurons. Further study on the mechanisms about the combine effects of supplementation of DHA and energy restriction on brain function is urgently needed in this area.

Keywords: Docosahexaenoic acid, Brain function, N-3 fatty acid deficiency, Prolonged fasting, Endurance physical exercise, Brain-derived neurotropic factor

\section{INTRODUCTION}

It was revealed that hypoxia has a negative effect on brain function, however docosahexaenoic acid (DHA) and physical exercise has a positive effects through electroencephalography analysis (reduced the delta activity). DHA (22:6n-3) is a vital component of phospholipids in cellular membranes and especially concentrated in mammalian nervous system. In addition, DHA plays an important role in neural and retinal development of infant during perinatal and early postnatal periods (Neuringer et al., 1988; Yamamoto et al., 1987). Losses in neural function in $\mathrm{n}-3$ fatty acid deficiency have been associated with decreased brain DHA and increased brain docosapentaenoic acid (DPAn-6, 22:5n-6) contents (Neuringer et al., 1988; Okuyama et al., 1996; Salem et al.,
2001). Since there is an inadequate amount of 22-carbom polyunsaturated fatty acids (PUFAs) during critical brain development period in $\mathrm{n}-3$ fatty acid deficient condition, it is a possible hypothesis that feeding DPA would prevent loss in neural function in n-3 fatty acid deficiency. Thus this review will describe the biological consequences of losses in brain DHA using a first generation model. In addition, it will present whether there is any benefit of DPAn-6 supplementation in nervous function in n- 3 fatty acid deficiency.

And also, it was well known that prolonged fasting (PF) and energy restriction with endurance physical exercise (EX) stimulate cognitive function and brain function, which is caused by upregulated ketone body and upregulated brain-derived neurotropic factor (BDNF) responses. DHA-diet supplementation reduced the

\footnotetext{
*Corresponding author: Sun Young Lim (i) https://orcid.org/0000-0002-8091-3496 Division of Marine Bioscience, Korea Maritime and Ocean University, 727 Taejong-ro, Yeongdo-gu, Busan 49112, Korea

Tel: +82-51-410-4757, Fax: +82-51-404-4750, E-mail: sylim@kmou.ac.kr Received: June 11, 2018 / Accepted: July 23, 2018
}

This is an Open Access article distributed under the terms of the Creative Commons Attribution Non-Commercial License (http://creativecommons.org/licenses/by-nc/4.0/) which permits unrestricted non-commercial use, distribution, and reproduction in any medium, provided the original work is properly cited. 
markers of muscle damage, inflammation caused by increased prostaglandin levels following acute physical exercise. And also, DHA supplementation has a positive effect on muscle damage, muscle function as well as brain function (Ochi and Tsuchiya, 2018).

However, we still do not know the exact mechanism about the brain function after EX and DR. Therefore, this study helps to clarify the mechanisms about the combined impacts of DHA (22:6n-3), EX and PF on the brain function. In this study, we analyze on two main topics: 'DHA diet and brain function' and 'physical exercise and brain function.'

\section{EFFECT OF DOCOSAHEXAENOIC ACID DIET ON BRAIN FUNCTION}

Essential fatty acids are not synthesized in the body and must be consumed by dietary foods. They are divided into two classes, linoleic acid (18:2n-6, LA) or linolenic acid (18:3n-3, LNA). LA is incorporated in arachidonic acid (20:4n-6, AA) and DPAn-6, while LNA is synthesized into eicosapentaenoic acid (20:5n-3, EPA) and DHA in the body. DHA, a long chain PUFA is highly enriched in the membrane phospholipids of the brain, neuronal tissue and retina (Neuringer et al., 1988; Yamamoto et al., 1987).

Studies on interactions between essential fatty acids and nervous system function have been conducted in the field of lipid and essential fatty acid. Moreover, there are marked efforts to determine the effects of essential fatty acids on growth and development in the nervous system. Since 1970, many studies have demonstrated that a dietary deficiency in $\mathrm{n}-3$ fatty acids led to a loss in nervous system DHA (Neuringer et al., 1988; Okuyama et al., 1996; Salem et al., 2001; Tinoco et al., 1979) and that loss of brain DHA was highly associated with visual and cognitive defects as observed in animal and human infant studies (Bourre et al., 1989; Catalan et al., 2002; Greiner et al., 2001; Moriguchi et al., 2000; Neuringer et al., 1984; Neuringer et al., 1986; Pawlosky et al., 1997; Wainwright et al., 1998; Wheeler et al., 1975; Yamamoto et al., 1987). When the level of DHA fell in the brain and retina, there was a concomitant increase in the $\mathrm{n}-6$ docosapentaenoic acid (DPAn-6) (Mohrhauer and Holman, 1963; Tinoco et al., 1978). At this point, there are two important issues; one issue is whether the animal models can generate large enough losses in nervous system DHA so that functional changes can be observed. Second question is whether increased brain DPAn-6 can replace with nervous function of brain DHA in the $\mathrm{n}-3$ deficiency.

Typically those animal studies have been accomplished through the use of a two generational model in which the dam is deprived of $n-3$ fatty acid sources and her offspring are then weaned to the same deficient diet. Later artificial rearing methods have been developed to allow for the more rapid induction of a brain DHA deficiency in the newborn rat and is more similar to the case of human nutrition (Lim et al., 2005a; Moriguchi et al., 2004; Ward et al., 1996). Lim et al. (2005b) developed an aritifical rearing method and produced animals with $70 \%$ loss in brain DHA in the first generation. The first trimester after birth is a very crucial period in which a dietraty DHA supply was needed for proper brain funtions. This sutdy measured spatial task performance of those $n-3$ fatty acid deficient animals, suggeting that the DPAn- 6 fed animals performed no better than animals fed only LA, suggesting that dietary DPAn-6 produced none of the benefit afforded by DHA addition. This finding is highly associated with an observation that the brain fatty acid profiles at adulthood were identical between the LA and DPA groups. Given that these molecules only differ by a single double bond at the $\mathrm{n}-3$ position on the chain, it is relevant to consider what mechanisms may be responsible for the difference in brain function. NMR studies of DHAphospholipids also indicate a highly flexible molecule with rapid transitions between a large number of conformers (Gawrisch et al., 2003). Eldho et al. (2003) compared the difference of DHA containing phospholipids to species containing DPAn-6 in lipid matrix properties and observed that DHA in the methyl end is more flexible and isomerizes with shorter correlation times such that interactions with membrane proteins would be altered. Take together, these mechanisms are largely derived through DHA incorporation in place of other fatty acids into membrane receptor function, neuronal apoptosis, biophysical property, biological membranes of phospholipids and cell signaling mechanisms.

Moriguchi and Salem (2003) investigated the recovery of spatial task performance as DHA was replenished in the brain and found that the escape latency became significantly different from the DHA group in a group where the brain DHA was $7.6 \%$ but not different in a group where brain DHA was $10.6 \%$. Lim et al. (2005b) also investigated the effect of supplementation with DHA into n-3 fatty acid deficient diet on improvement of learning related brain function and found that supplementation with DHA into the $\mathrm{n}-3$ fatty acid deficient diet improved the spatial learning ability in rats as assessed by Morris water maze test. Thus the significance of these findings is that at least some of the adverse effects of DHA deficiency during neurodevelopment may be reversible with an $\mathrm{n}-3$ fatty acid supplemented diet. 


\section{EFFECT OF EXERCISE, ENDURANCE PHYSICAL EXERCISE, AND PROLONGED FASTING ON BRAIN FUNCTION}

It was well reported that regular physical exercise improves memory function by increasing neurogenesis in the hippocampus, and it is also reported that circadian rhythms modulate synaptic plasticity in the hippocampus during exercise. This results shows that the day exercise much helper to the brain function than in the night or dawn exercise (So et al., 2017). It was related mechanisms of several factors such as enhanced or decreased AMP-activated protein kinase (AMPK), BDNF, insulin-like growth factor 1 (IGF-1) levels following regulatory training or physical exercise. This is called as neurotrophic factors. It is understood and reported that regular exercise increased brain function by IGF-1, BDNF, and AMPK activation in healthy people and even in type 1 diabetes mellitus (T1DM) (Tonoli et al., 2015).

In the hypothalamus, BDNF suppress food intake and increase energy expenses. This is why regular exercise and endurance exercise with energy depletion promote synaptic plasticity and neuronal genesis in the brain. Actually, BDNF plays an important portion in the unification and optimization of behavioral and metabolic responses to circumstances with restricted energy sources and severe athletic competition. BDNF is considered as a key controller of cognitive function, and also acting on brain metabolism and even cardiovascular health. In some aspects, BDNF regulation by food ingestion is linked with the capacity of neurotrophin to adjust peripheral energy metabolism. This means reduced blood BDNF levels are noted in obese people and also in the type 2 diabetes mellitus (T2DM) (Rothman et al., 2012), whereas they have different mechanism. That is why decreased BDNF status results in hyperphagia and obesity.

Actually, obesity is affected with the changes of leptin, insulin, and corticosterone with food uptake and physical activity amounts. It was generally believed that rotating blood leptin enters the brain barrier and interplays with neurons in the hypothalamus to repress appetite. It might be revealed as BDNF is related mechanisms with the leptin and insulin mechanisms. It is also related mechanism with the serotonin levels during exercise, especially in the outdoor exercise with sunshine. It was also known that serotonin controls the leptin sensitivity following physical exercise. That's because obese sedentary people are at danger for cardiovascular problems, hypertension, T2DM and even neurological diseases. So, it is well known that valuable effects of physical exercise and any kinds of EX or PF on brain structural plasticity and behaviors identified
(Rothman et al., 2012).

EX or energy restriction (focus on the ketone body) on brain function also proposed our previous researches (Park and Kwak, 2017). It is also reported that ER stimulate the brain region with the change of gene expression encoding proteins engaged in neuronal synaptic plasticity, neurotrophic signalling factors, and cellular stress resistance (Alirezaei et al., 2011).

EX also stimulate neurogenesis, synaptogenesis, synaptic plasticity and therefore increase cognitive function and motor function. It is also increase insulin sensitivity, fatty acid mobilization, ketone body production and decrease blood pressure and even inflammation.

It is almost the same effects with the physical exercise on cognitive function besides mitochondrial biogenesis.

Actually, DR stimulates cell protective responses in nearly all tissues and organs including brain. And also it activates living gene expressions and lengthens lifestyle in a fashion of conserved state. For the human beings, dietary restriction studies reveal the novel tactics to lengthen health span and prevent age-related diseases and disorders. Interestingly, even exogenuous ketone improved exercise performance time, cognitive function in rats and also it can help to treat in T2DM patients (Prehn et al., 2017) and neurodegenerative diseases, such as Alzheimer diseases and Parkinson diseases. In this mechanism, BDNF has also shown to play prominent role in this mechanism like physical exercise. Prolonged physical activity and EX or DR stimulate ketone body. Upregulated BDNF can activate mitochondrial biogenesis to elevate neuronal bioenergetics and enable synaptic formation. And it also can activate DNA repair in neurons.

\section{CONCLUSIONS}

Highly polyunsaturated fatty acids including DHA and AA are enriched in the membrane phospholipids of the nervous system and are known to play an important role in the nervous function. When the level of DHA fell in the brain and retina, there was a concomitant increase in the $\mathrm{n}-6$ docosapentaenoic acid (DPAn-6). Since brain DPAn-6 dose not substitute with the function of brain DHA in n-3 fatty acid deficiency, it is very important that proper DHA levels in the nervous system are maintained for optimal brain and retina function. BDNF has also shown to play prominent role in this mechanism like physical exercise. Prolonged physical activity and EX, PF or DR stimulate ketone body. It is revealed that ketone body also stimulate BDNF. Upregulated BDNF can activate mitochondrial biogenesis to elevate neuronal 
bioenergetics and enable synaptic formation. And it also can activate DNA repair in neurons. Further study on the mechanisms about the combine effects of supplementation of DHA and EX, $\mathrm{PF}$, and $\mathrm{DR}$ on brain function is urgently needed in this area.

\section{CONFLICT OF INTEREST}

No potential conflict of interest relevant to this article was reported.

\section{ACKNOWLEDGMENTS}

This research was supported by Basic Science Research Program through the National Research Foundation of Korea (NRF) funded by the Ministry of Science, ICT and Future Planning (NRF2017R1A2B4005915).

\section{REFERENCES}

Alirezaei M, Kemball CC, Whitton JL. Autophagy, inflammation and neurodegenerative disease. Eur J Neurosci 2011;33:197-204.

Bourre JM, Francois M, Youyou A, Dumont O, Piciotti M, Pascal G, Durand $\mathrm{G}$. The effects of dietary alpha-linolenic acid on the composition of nerve membranes, enzymatic activity, amplitude of electrophysiological parameters, resistance to poisons and performance of learning tasks in rats. J Nutr 1989;119:1880-1892.

Catalan J, Moriguchi T, Slotnick B, Murthy M, Greiner RS, Salem N Jr. Cognitive deficits in docosahexaenoic acid-deficient rats. Behav Neurosci 2002;116:1022-1031.

Eldho NV, Feller SE, Tristram-Nagle S, Polozov IV, Gawrisch K. Polyunsaturated docosahexaenoic vs docosapentaenoic acid-differences in lipid matrix properties from the loss of one double bond. J Am Chem Soc 2003;125:6409-6421.

Gawrisch K, Eldho NV, Holte LL. The structure of DHA in phospholipid membranes. Lipids 2003;38:445-452.

Greiner RS, Moriguchi T, Slotnick BM, Hutton A, Salem N. Olfactory discrimination deficits in n-3 fatty acid-deficient rats. Physiol Behav 2001; 72:379-385.

Lim SY, Hoshiba J, Moriguchi T, Salem N Jr. N-3 fatty acid deficiency induced by a modified artificial rearing method leads to poorer performance in spatial learning tasks. Pediatr Res 2005a;58:741-748.

Lim SY, Hoshiba J, Salem N. An extraordinary degree of structural specificity is required in neural phospholipids for optimal brain function: n-6 docosapentaenoic acid substitution for docosahexaenoic acid leads to a loss in spatial task performance. J Nerurochem 2005b;95:848-857.
Mohrhauer H, Holman RT. Alteration of the fatty acid composition of brain lipids by varying levels of dietary essential fatty acids. J Neurochem 1963;10:523-530.

Moriguchi T, Greiner RS, Salem N Jr. Behavioral deficits associated with dietary induction of decreased brain docosahexaenoic acid concentration. J Neurochem 2000;75:2563-2573.

Moriguchi T, Lim SY, Greiner R, Lefkowitz W, Loewke J, Hoshiba J, Salem N Jr. Effects of an n-3-deficient diet on brain, retina, and liver fatty acyl composition in artificially reared rats. J Lipid Res 2004;45:14371445.

Moriguchi T, Salem N Jr. Recovery of brain docosahexaenoate leads to recovery of spatial task performance. J Neurochem 2003;87:297-309.

Neuringer M, Anderson GJ, Connor WE. The essentiality of n-3 fatty acids for the development and function of the retina and brain. Annu Rev Nutr 1988;8:517-541.

Neuringer M, Connor WE, Lin DS, Barstad L, Luck S. Biochemical and functional effects of prenatal and postnatal omega 3 fatty acid deficiency on retina and brain in rhesus monkeys. Proc Natl Acad Sci U S A 1986;83:4021-4025.

Neuringer M, Connor WE, Van Petten C, Barstad L. Dietary omega-3 fatty acid deficiency and visual loss in infant rhesus monkeys. J Clin Invest 1984;73:272-276.

Ochi E, Tsuchiya Y. Eicosahexanoic acid (EPA) and docosahexanoic acid (DHA) in muscle damage and function. Nutrients 2018;10(5):552.

Okuyama H, Kobayashi T, Watanabe S. Dietary fatty acids - the N-6/N-3 balance and chronic elderly diseases. Excess linoleic acid and relative N-3 deficiency syndrome seen in Japan. Prog Lipid Res 1996;35:409457.

Park CH, Kwak YS. Analysis of energy restriction and physical activity on brain function: the role of ketone body and brain-derived neurotrophic factor. J Exerc Rehabil 2017;13:378-380.

Pawlosky RJ, Denkins Y, Ward G, Salem N Jr. Retinal and brain accretion of long-chain polyunsaturated fatty acids in developing felines: the effects of corn oil-based maternal diets. Am J Clin Nutr 1997;65:465-472.

Prehn K, Jumpertz von Schwartzenberg R, Mai K, Zeitz U, Witte AV, Hampel D, Szela AM, Fabian S, Grittner U, Spranger J, Flöel A. Caloric restriction in older adults-differential effects of weight loss and reduced weight on brain structure and function. Cereb Cortex 2017;27: 1765-1778.

Rothman SM, Griffioen KJ, Wan R, Mattson MP. Brain-derived neurotrophic factor as a regulator of systemic and brain energy metabolism and cardiovascular health. Ann N Y Acad Sci 2012;1264:49-63.

Salem N Jr, Litman B, Kim HY, Gawrisch K. Mechanisms of action of docosahexaenoic acid in the nervous system. Lipids 2001;36:945-959.

So JH, Huang C, Ge M, Cai G, Zhang L, Lu Y, Mu Y. Intense exercise pro- 
motes adult hippocampal neurogenesis but not spatial discrimination. Front Cell Neurosci 2017;11:13.

Tinoco J, Babcock R, Hincenbergs I, Medwadowski B, Miljanich P. Linolenic acid deficiency: changes in fatty acid patterns in female and male rats raised on a linolenic acid-deficient diet for two generations. Lipids 1978;13:6-17.

Tinoco J, Babcock R, Hincenbergs I, Medwadowski B, Miljanich P, Williams MA. Linolenic acid deficiency. Lipids 1979;14:166-173.

Tonoli C, Heyman E, Buyse L, Roelands B, Piacentini MF, Bailey S, Pattyn $\mathrm{N}$, Berthoin S, Meeusen R. Neurotrophins and cognitive functions in T1D compared with healthy controls: effects of a high-intensity exercise. Appl Physiol Nutr Metab 2015;40:20-27.

Wainwright PE, Xing HC, Girard T, Parker L, Ward GR. Effects of dietary n-3 fatty acid deficiency on morris water-maze performance and amphetamine-induced conditioned place preference in rats. Nutr Neurosci 1998;1:281-293.

Ward G, Woods J, Reyzer M, Salem N Jr. Artificial rearing of infant rats on milk formula deficient in n-3 essential fatty acids: a rapid method for the production of experimental n-3 deficiency. Lipids 1996;31:7177.

Wheeler TG, Benolken RM, Anderson RE. Visual membranes: specificity of fatty acid precursors for the electrical response to illumination. Science 1975;188:1312-1314.

Yamamoto N, Saitoh M, Moriuchi A, Nomura M, Okuyama H. Effect of dietary $\alpha$-linolenate/linoleate balance on brain lipid compositions and learning ability of rats. J Lipid Res 1987;28:144-151. 